\title{
Study on Effect of Aerodynamic Configuration on Aerodynamic Performance of Mars Ascent Vehicles
}

\author{
Qi Li, ${ }^{1}$ Wu Yuan, ${ }^{2}$ Rui Zhao, ${ }^{3}$ and Haogong Wei $\mathbb{D}^{1}$ \\ ${ }^{1}$ Beijing Institute of Spacecraft System Engineering, CAST, Beijing, China 100094 \\ ${ }^{2}$ Computer Network Information Center, Chinese Academy of Sciences, Beijing, China 100190 \\ ${ }^{3}$ School of Aeronautics and Astronautics, Beijing Institute of Technology, Beijing, China 100081
}

Correspondence should be addressed to Qi Li; qi-ge-ge@163.com

Received 10 August 2021; Accepted 29 November 2021; Published 29 January 2022

Copyright ( 2022 Qi Li et al. Exclusive Licensee Beijing Institute of Technology Press. Distributed under a Creative Commons Attribution License (CC BY 4.0).

\begin{abstract}
The Mars surface take-off and ascent technology is one of the key technologies for realizing the Mars sample return mission. Different from that on the moon, the gravity acceleration on the surface of Mars is $3.71 \mathrm{~m} / \mathrm{s}^{2}$, so that the gravity loss is larger than that on the moon; a rarefied atmosphere is found on the surface of Mars, and although it is only about 1\% of the Earth's atmosphere, its effect on aerodynamic drag in the process of ascent shall also be considered. In this paper, the aerodynamic performance demand of ascent vehicles is analyzed in light of the mission requirements for take-off and ascent from the surface of Mars. Based on the results of literature research and supersonic CFD static simulation, the influence of forebody and afterbody shapes of ascent vehicles on aerodynamic drag and static stability is studied, respectively. The forebody shape of ascent vehicles with better aerodynamic performance is proposed, and the subsequent improvement direction of aerodynamic configuration is clarified, providing necessary theoretical and data support for the aerodynamic selection of Mars ascent vehicles.
\end{abstract}

\section{Introduction}

According to the white paper, "China's Space Activities in 2016" [1], the Mars sample return mission represents one of the main tasks to be implemented in China's deep space exploration field in the next 10 years. As the key technologies to be developed for Mars sample return, the design, analysis, and verification for Mars take-off and ascent can play a very important support role in the engineering design and implementation of the rover. Moreover, the shape design of Mars ascent vehicles (MAV for short) is the key link of the Mars take-off and ascent technology, which has an important impact on the design of the power system, attitude control system, structure, and loading system.

In accordance with the published literature, countries all over the world mainly adopt two routes for the shape design of Mars ascent vehicles. One is the slender body similar to the missile/rocket [2-4], and the other is the short blunt body with a high loading volume ratio $[5,6]$. The former is mainly developed for the solid propulsion system, while the latter is mainly developed for the liquid propulsion system.

The thickness of the Martian atmosphere is about $100 \mathrm{~km}$, and the atmospheric density at the same altitude is only $1 \% \sim 10 \%$ of the earth's atmosphere $[7,8]$. However, due to the severe quality restriction of the propulsion system, the speed loss of the MAV caused by the atmospheric resistance of Mars cannot be ignored. To minimize the velocity loss, save the fuel for the propulsion system, and improve safety during the ascent, we should strictly constrain and optimize the drag characteristics of the ascent vehicle.

According to a similar design abroad, the supersonic region is the region with the largest dynamic pressure and the most obvious velocity loss of the ascent vehicle during take-off and ascent from Mars [9]. Tang et al. [10] studied the influence of different slenderness ratio on MAV resistance characteristics based on the shape of slender body. Miao et al. [11] studied the resistance variation characteristics of a variety of rotating warheads with different 
generatrix lines. According to the author's preliminary study, it is found that the influence of the forebody generatrix of slender body and short blunt body on the resistance characteristics is not consistent.

In this paper, the RANS numerical simulation method is used to calculate and analyze the aerodynamic characteristics of two types of MAV, i.e., slender body and short blunt cone cylinder. The influence law and efficiency of the change of forebody generatrix parameters on the aerodynamic performance of different types of Mars risers are explored, which can provide design basis and data basis for the aerodynamic selection of MAV.

\section{Aerodynamic Performance Demands of MAV}

2.1. Drag Performance. The ascent vehicle mainly relies on the main engine to provide the power, and the velocity loss caused by aerodynamic drag, gravity, and other factors in the process of ascent directly affects the design of a propulsion system. In light of the rarefied atmosphere on Mars, the aerodynamic loss on the Mars surface during take-off and ascent is much smaller than that on the Earth during launch. However, according to the previous analysis, the velocity loss caused by aerodynamic drag accounts for $18 \%$ of the total velocity loss for a short blunt ascent vehicle, while it accounts for about $6.67 \%$ in the total velocity loss for a slender ascent vehicle, both of which cannot be ignored. Therefore, the aerodynamic drag of Mars ascent vehicles still needs to be reduced.

Through the preliminary aerodynamic configuration design and drag performance budget, the optimization indicators for the drag performance of ascent vehicles are proposed:

(1) When the maximum windward section is taken as the reference area, the zero-attack-angle drag coefficient of an ascent vehicle with a short blunt body shall not be higher than 1.02 at Ma2.0 and 0.8 at Ma4.1, respectively

(2) When the maximum windward section is taken as the reference area, the zero-attack-angle drag coefficient of an ascent vehicle with a slender body shall not be higher than 0.9 at Ma2.0 and 0.44 at Ma4.1, respectively

2.2. Static Stability. For the ascent vehicle with a smalltorque attitude control system, its static stability within the main flight attitude range is indispensable. If the ascent vehicle is statically unstable in trimmed flight, a little disturbance such as crosswinds and asymmetric jets will cause the ascent vehicle to deviate from the designed trimmed attitude and result in large attitude drift, thereby increasing aerodynamic drag and inducing large oscillation. The attitude drift caused by static instability can be resisted only when the thrust of the attitude control system is large enough, but the large thrust will also lead to the overall increase in dry weight of the control system and additional consumption of propellant. Therefore, the ascent vehicle should be capable of static stability in the atmosphere.

Similar to rockets, missiles, and other flight vehicles, the static stability of ascent vehicles can be characterized by the relative position between the center of mass and the center of pressure on the centroid axis. When the center of pressure is behind the center of mass, namely, $(\mathrm{Xcp}-\mathrm{Xcg})>0$, the ascent vehicle will be statically stable; otherwise, it will be statically unstable; the shorter distance between the center of pressure and the center of mass indicates the lower static stability. Generally, (Xcp-Xcg)/L characterizes the static stability of flight vehicles, and $L$ is the reference length of a flight vehicle. According to the design criteria for missiles, (Xcp-Xcg)/L is generally required to be between 0.03 and 0.06 for normal or tailless missiles [12]. If the static stability is too high, the maneuverability will be poor, and it will be difficult to adjust the flight trajectory by changing the flight attitude through the rudder surface or RCS jets; if the static stability is too small, the antidisturbance ability will be poor, and additional resources will be required for ensuring the trimmed flight.

To sum up, the static stability of Mars ascent vehicles can be achieved by aerodynamic configuration, mass configuration, and the attitude control system by reference to the design ideas of rockets or missiles.

\section{Selection of Shape Parameters of the Forebody of MAV}

During the ascent of an ascent vehicle from the surface of Mars, its aerodynamic drag mainly comes from shock wave drag, wall friction drag, and pressure drag and is proportional to the inflow pressure. The preliminary trajectory analysis reveals that the maximum dynamic pressure during the ascent of the vehicle occurs when Ma is between 1.5 and 4.5, which is the supersonic region. According to the previous research, the shock wave drag of a flight vehicle in the supersonic region accounts for more than $70 \%$ of the total drag [10]. Therefore, reducing the shock wave drag of the ascent vehicle is crucial to the lower energy consumption and system cost.

Since the ascent vehicle mainly flies at a small attack angle during ascent, its shock wave drag is mainly caused by the nose shock. Therefore, the supersonic shock wave drag can be significantly reduced by controlling the forebody shape of the ascent vehicle and changing the direction of shock force, and this method has good engineering applicability. For example, in the supersonic aerospace vehicle demonstrator X-51A launched in 2010, the forebody shape is optimized. The wave rider configuration is adopted, which ensures that when the vehicle passes through the air at a hypersonic velocity, the sharp cone weakens the strength of normal shock wave, and all the pressure generated by the shock wave system is directly applied under the body to provide the lift so that the supersonic aerodynamic drag of the vehicle is greatly reduced [13].

With the aerodynamic configuration design of the warhead as a reference, the shape of the warhead is determined by the generatrix curve type. With the theoretical vertex of the cone as the coordinate origin, the $x$-axis is along the symmetry axis of the projectile and points to the bottom of the projectile, and $r$ is the radius of the revolutionary body. As for the five common generatrix curve types, namely, spherical-conical, circular arc, parabolic, exponential, and von Karman curves, the equations can be written as follows [13]: 


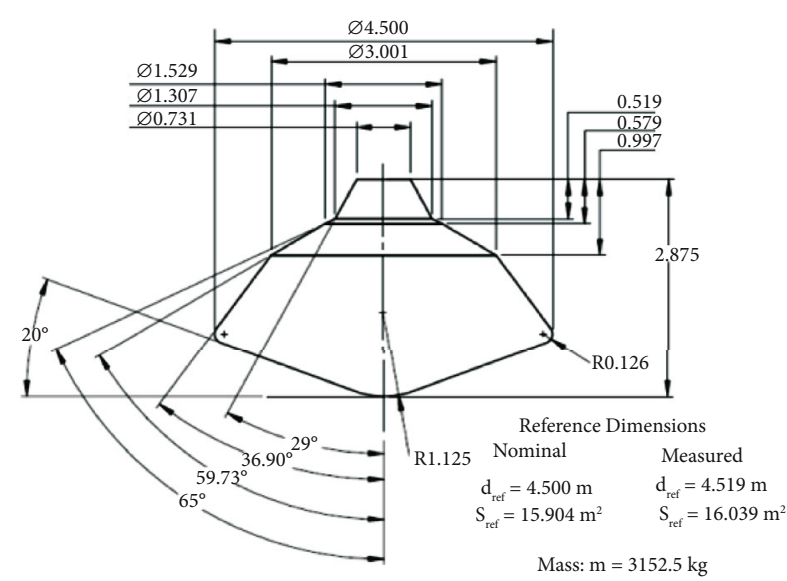

(a)

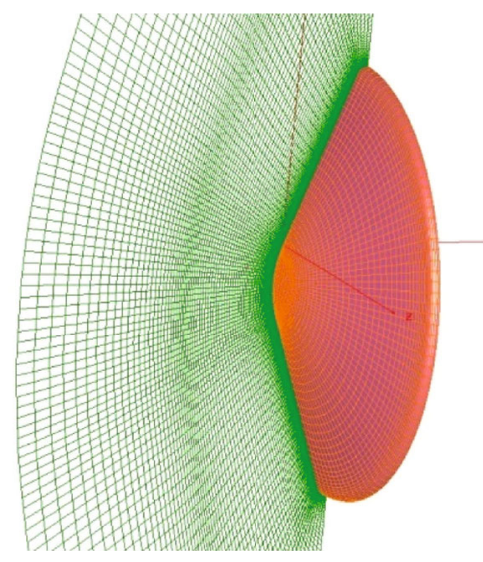

(b)

FIGURE 1: Aerodynamic shape and grid diagram of forebody of Mars Laboratory (MSL). (a) Main body dimension parameters of MSL. (b) Grid representation of forebody.

TABLE 1: Incoming flow conditions for MSL supersonic aerodynamic calculation.

\begin{tabular}{cccccc}
\hline$M_{\infty}$ & $\alpha\left(^{\circ}\right)$ & $V_{\infty}(\mathrm{m} / \mathrm{s})$ & $\rho_{\infty}\left(\mathrm{kg} / \mathrm{m}^{3}\right)$ & $T_{\infty}(\mathrm{K})$ & $\gamma_{\mathrm{eff}}$ \\
\hline 2.09 & $\begin{array}{c}0,6,11, \\
16,20,24\end{array}$ & 472 & $4.634 E-03$ & 190.2 & 1.24822 \\
\hline
\end{tabular}

(1) Spherical-conical shape: $r=\left(R_{d} / L\right) x$

(2) Circular arc shape: $r=\rho\left[\sqrt{1-((L-x) / R)^{2}}-1\right]+R_{d}$

(3) Parabolic shape: $r=\left(R_{d} / L\right)\left(2 x-\left(x^{2} / L\right)\right)$

(4) Exponential shape: $r=R_{d}(x / L)^{n}$

(5) von Karman curve shape: $r=\left(R_{d} / \sqrt{\pi}\right)$ $\sqrt{\varphi-(1 / 2) \sin 2 \varphi}, \varphi=\arccos (1-2(x / L))$

where $L$ is the theoretical length of the curve segment, $R_{d}$ is the maximum radius of the curve segment, $\rho$ is the curvature radius of the arc curve, and $n$ is the exponent of the exponential curve, which can be taken between 0 and 1 . When $n=1$, it is a cone.

\section{Description and Verification of the Algorithm}

4.1. Algorithm Description. Three-dimensional compressible viscous gas dynamic equations are used as the governing equations of flow field. The governing equation is expressed as follows:

$$
\frac{\partial}{\partial t} \int_{\Omega} \mathbf{Q} d V+\int_{\partial \Omega} \mathbf{F} \cdot \widehat{\mathbf{n}} d S=\int_{\partial \Omega} \mathbf{G} \cdot \widehat{\mathbf{n}} d S,
$$

where $\Omega$ is the control body, $\partial \Omega$ is the control surface, $\mathbf{Q}$ is the conservation variable vector, $\mathbf{F}$ is the convection term, and $\mathbf{G}$ is the viscous diffusion term.
$\mathbf{Q}=\left(\begin{array}{c}\rho \\ \rho u \\ \rho v \\ \rho w \\ E\end{array}\right)$,

$\mathbf{F}=\left(\begin{array}{c}\rho u \\ \rho u^{2}+p \\ \rho u v \\ \rho u w \\ u(E+p)\end{array}\right) \widehat{\mathbf{i}}+\left(\begin{array}{c}\rho v \\ \rho v u \\ \rho v^{2}+p \\ \rho v w \\ v(E+p)\end{array}\right) \widehat{\mathbf{j}}+\left(\begin{array}{c}\rho w \\ \rho w u \\ \rho w v \\ \rho w^{2}+p \\ w(E+p)\end{array}\right) \widehat{\mathbf{k}}$,

$\mathbf{G}=\left(\begin{array}{c}0 \\ \tau_{x x} \\ \tau_{x y} \\ \tau_{x z} \\ u \tau_{x x}+v \tau_{x y}+w \tau_{x z}-q_{x}\end{array}\right)$

$$
\begin{aligned}
& \widehat{\widehat{\mathbf{i}}}+\left(\begin{array}{c}
0 \\
\tau_{y x} \\
\tau_{y y} \\
\tau_{y z} \\
u \tau_{y x}+v \tau_{y y}+w \tau_{y z}-q_{y}
\end{array}\right) \\
& \cdot \widehat{\mathbf{j}}+\left(\begin{array}{c}
0 \\
\tau_{z x} \\
\tau_{z y} \\
\tau_{z z} \\
u \tau_{z x}+v \tau_{z y}+w \tau_{z z}-q_{z}
\end{array}\right) \widehat{\mathbf{k}}
\end{aligned}
$$

In the above formula, $\rho$ is the density, $p$ is the pressure, $u, v$, and $w$ are velocities in three directions, and $\mathbf{E}$ is total energy. 


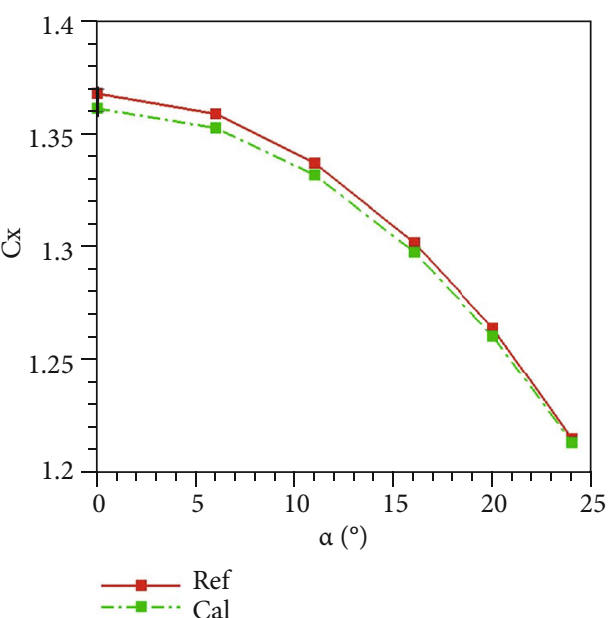

(a)

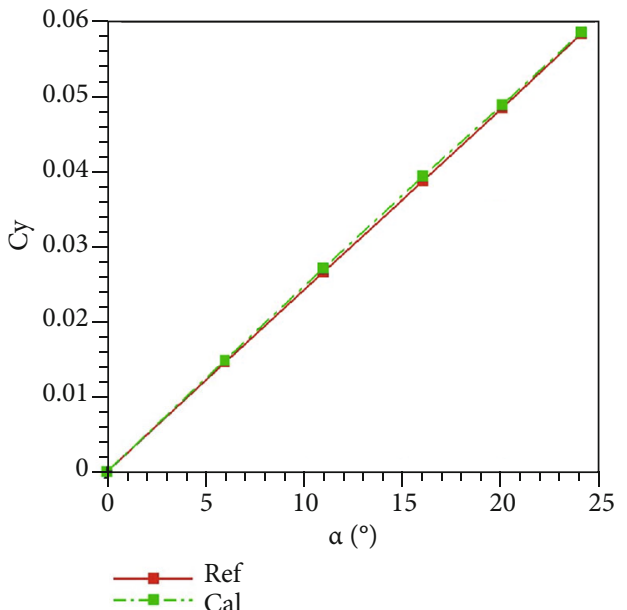

(b)

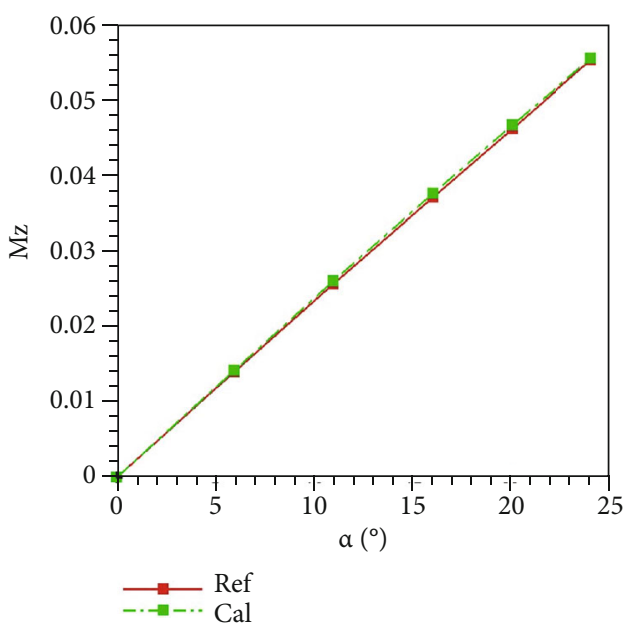

(c)

Figure 2: Comparison between the calculated data of Mars Laboratory (MSL) aerodynamics and the literature data. (a) Axial force coefficient. (b) Normal force coefficient. (c) Pitching moment coefficient.

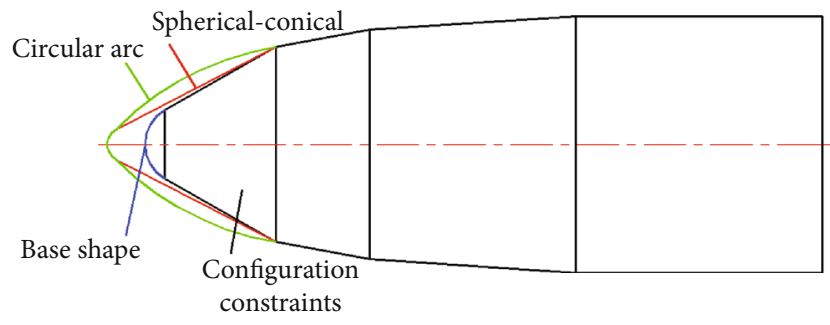

Figure 3: Comparison between the basic configuration and the improved spherical-conical and circular arc shapes of the slender ascent vehicle.

There are

$$
\begin{aligned}
E & =\frac{p}{\gamma-1}+\frac{1}{2} \rho\left(u^{2}+v^{2}+w^{2}\right) \\
\boldsymbol{\tau} & =\mu\left[\begin{array}{ccc}
2 u_{x} & u_{y}+v_{x} & u_{z}+w_{x} \\
u_{y}+v_{x} & 2 v_{y} & v_{z}+w_{y} \\
u_{z}+w_{x} & v_{z}+w_{y} & 2 w_{z}
\end{array}\right]-\frac{2}{3} \mu\left(u_{x}+v_{y}+w_{z}\right) \mathbf{I} .
\end{aligned}
$$

In this paper, the finite volume method of grid center based on structural grid was used to solve the above governing equations. Among them, Roe's FDS scheme [14] was used to discretize the flow term, and MUSUL interpolation and Min-mod limiter were used to obtain the second-order accuracy. Term was iterated in LU-SGS format. The turbulent model of viscous diffusion term adopts an equation model based on SA [15].

4.2. Verification of an Example. The aerodynamic parameters of windward outsole supersonic in Mars Science Laboratory (MSL for short) [16] are selected as an example in this paper and compared with the literature data. The geometric dimensions of MSL are shown in Figure 1(a). Because the strong expansion of the rear body has a weak influence on the force coefficient, only the windward outsole is taken for calculation. Because there is no sideslip in the calculation, half-mode calculation is adopted. The calculated total grid number is about 309,000, with 106 points in the normal direction, and the height of the first layer grid in the normal direction is $0.15 \mathrm{~mm}$. Figure 1(b) shows the grid distribution 


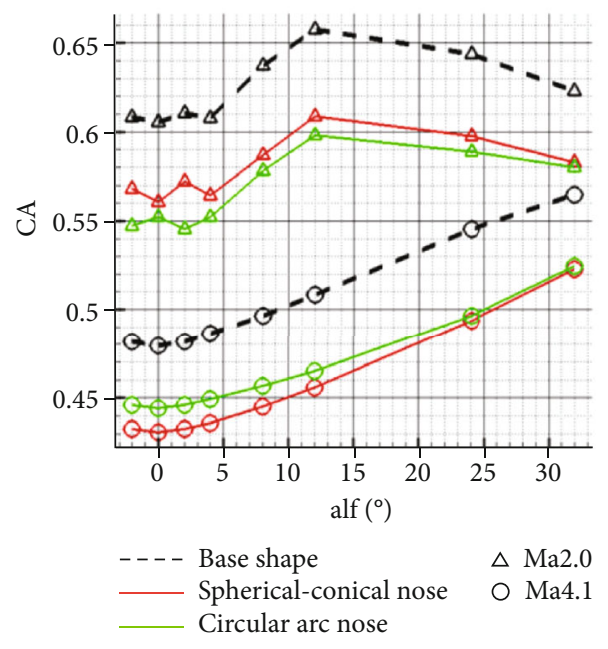

(a)

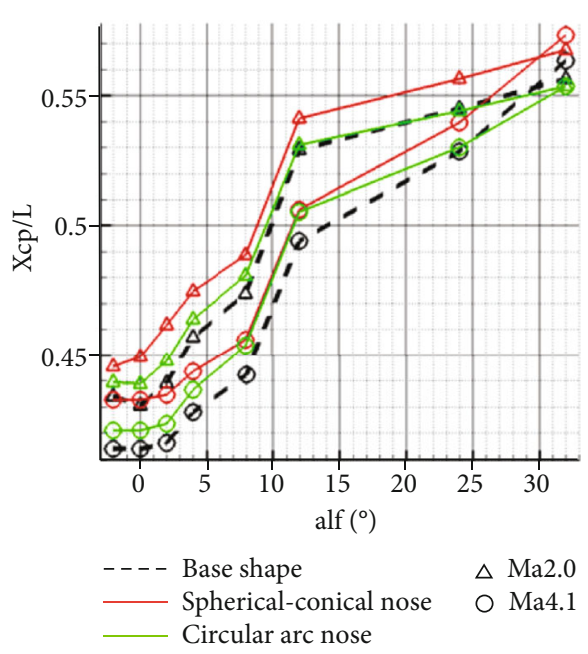

(b)

FIGURE 4: Comparison of supersonic aerodynamic characteristic curves between the basic shape and the improved spherical-conical and circular arc shapes of the slender ascent vehicle. (a) Axial force coefficient. (b) Pressure center coefficient.

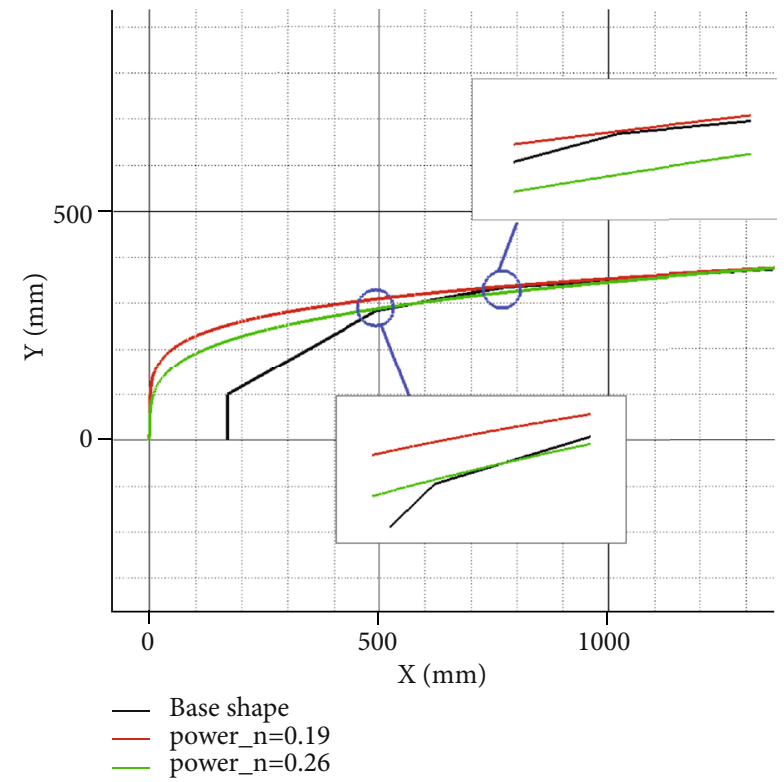

(a)

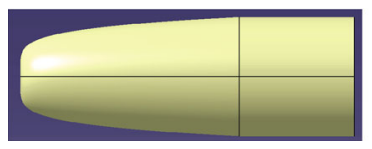

(b)

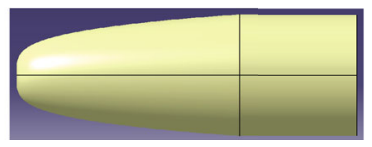

(c)
Figure 5: Optimization result of exponential noses of the slander ascent vehicle. (a) Comparison of optimized shape and configuration constraint contour of two exponential noses. (b) Exponential nose with $n=0.19$. (c) Exponential nose with $n=0.26$.

of symmetry plane and object plane of the windward outsole model.

The calculated inflow conditions are shown in Table 1, in which the local equivalent specific heat ratio of the atmospheric inflow from Mars is obtained by the formula $\gamma_{\text {eff }}=$ $((\eta+1) /(\eta-1))-\left(2 \eta /(\eta-1) \mathbf{M}_{\infty}^{2}\right)[17]$.
As shown in Figure 2, under the above calculation conditions, the aerodynamic coefficients obtained by the algorithm in this paper are consistent with the trend of literature results, and the relative deviation is less than $1 \%$. Therefore, the effectiveness of the aerodynamic algorithm used in this paper for calculating the aerodynamic characteristics of aircraft in the Martian atmosphere is verified.

\section{Calculation Result Analysis}

5.1. Influence Analysis of Aerodynamic Performance of Forebody Configuration of the Slender Ascent Vehicle. As shown in the figure below Figure 3, the Base shape is drawn first according to the minimum envelope contour of configuration constraints, in which the forebody is rounded and the radius of the bulbous is $115 \mathrm{~mm}$. Then, the sphericalconical and circular arc cones are designed, respectively. The theoretical length of $2.1 \mathrm{~m}$ is adopted for these two shapes; the contour line of the circular arc nose is the arc from the top of the spherical cone to the end of the third spherical-conical surface, and the radius is $848.7 \mathrm{~mm}$; the radius of both spherical-conical and arc noses is $50 \mathrm{~mm}$.

Based on CFD numerical simulation, the axial force coefficient and pressure center coefficient of the Base shape and the two improved shapes (namely, spherical-conical and circular arc noses) at Ma2.0 and Ma4.1 are obtained, as shown in Figure 4. By comparison, the following conclusions are obtained:

(1) After the Base shape is changed to the sphericalconical or the circular arc nose, the CA decreases slightly. The drag reduction effects of the two improved shapes are different at different $\mathrm{Ma}$, but the difference is subtle in general

(2) The zero-attack-angle CA of the spherical-conical nose is less than 0.58 at Ma2.0 and less than 0.44 at 


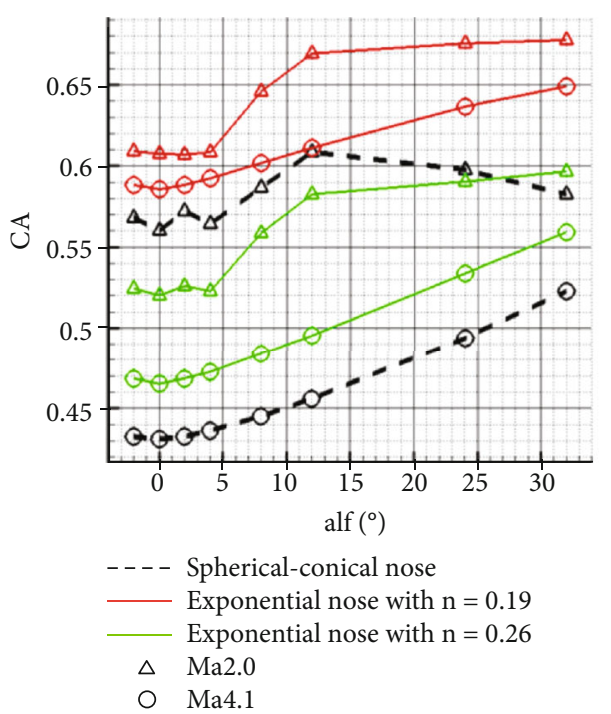

(a)

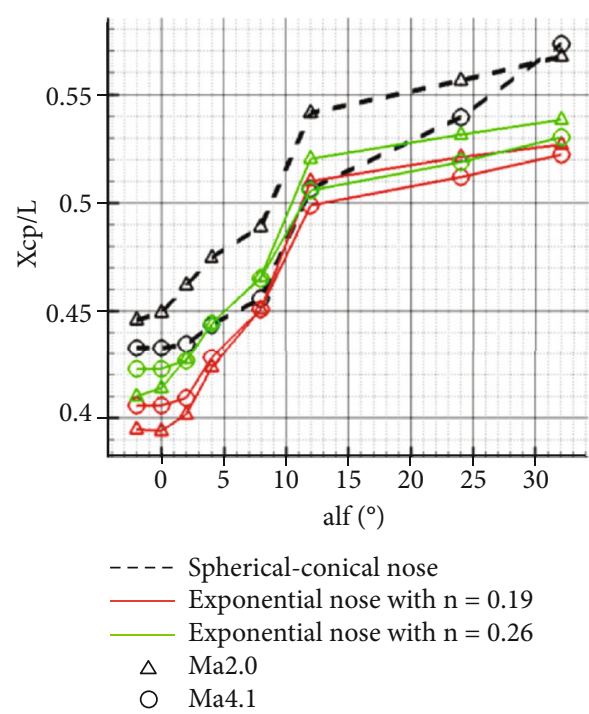

(b)

Figure 6: Comparison between supersonic aerodynamic characteristic curves of the spherical-conical nose and optimized exponential noses of the slender ascent vehicle. (a) Axial force coefficient. (b) Pressure center coefficient.

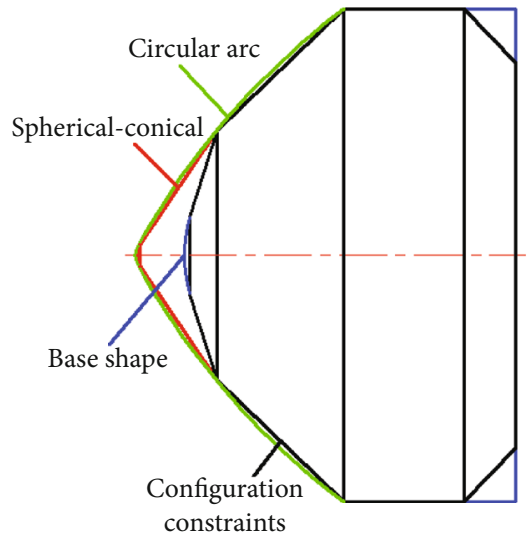

FIgURE 7: Comparison between the Base shape and improved spherical-conical and circular arc noses of the short blunt ascent vehicle.

Ma4.1, which can meet the requirements for optimized drag performance

(3) The pressure center coefficient of the Base shape and the two improved shapes is less than 0.45 at a small attack angle. The spherical-conical nose has the largest pressure center coefficient, but it is difficult to maintain static stability because the pressure center is too close to the nose

In addition to the spherical-conical and circular arc noses, this study also attempts to use the parabolic, von Karman, and exponential curves to optimize the nose of the slender ascent vehicle. However, the parabolic and von Karman curves cannot be generated because the configuration constraint contour cannot be broken through. Only the exponential curve can generate the blunt shape with a small $n$ value. The value of $n$ is set as 0.19 and 0.26 , respectively, and the latter has slightly deviated from the contour constraint, as shown in Figure 5.

Through CFD numerical simulation, the axial force coefficient and pressure center coefficient of spherical-conical and two optimized exponential noses at Ma2.0 and Ma4.1 are obtained, as shown in Figure 6. Comparative analysis reveals that as compared with that of the previous improved spherical-conical nose, the axial force of the two optimized exponential noses increases, while the pressure center coefficient decreases. Accordingly, the two optimized exponential noses fail to achieve the desired drag reduction and stability enhancement.

As indicated by the mechanism analysis, for the slender shape, the greater nose bluntness can result in the greater shock wave intensity and the stronger drag. In addition, the corner of the exponential nose with small $n$ is obvious, so that the pressure distribution on the surface changes rapidly. Therefore, the pressure center moves forward, and the slope increases with the attack angle. To sum up, the degradation of the stability can be predicted.

5.2. Influence Analysis of Aerodynamic Performance of Forebody Configuration of the Short Blunt Ascent Vehicle. The aerodynamic performance of the short blunt ascent vehicle is analyzed. As shown in Figure 7, firstly, the Base shape is drawn according to the minimum envelope contour of configuration constraints. In the figure, the nose is rounded, and the radius of bulbous is $336 \mathrm{~mm}$. Secondly, spherical-conical and circular arc noses are designed, respectively. The theoretical length of $1.0 \mathrm{~m}$ is adopted for these two shapes; the arc radius of the circular arc nose is $1990.6 \mathrm{~mm}$, and that of spherical-conical and circular arc noses is $50 \mathrm{~mm}$.

Through CFD numerical simulation, the axial force coefficient and pressure center coefficient of the Base shape and 


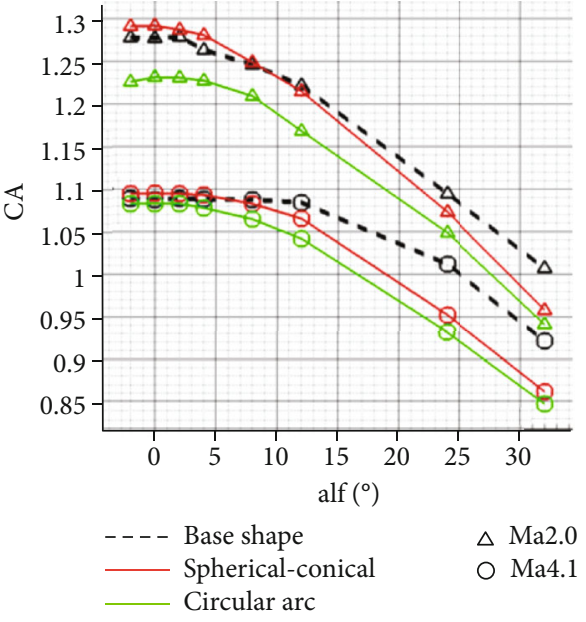

(a)

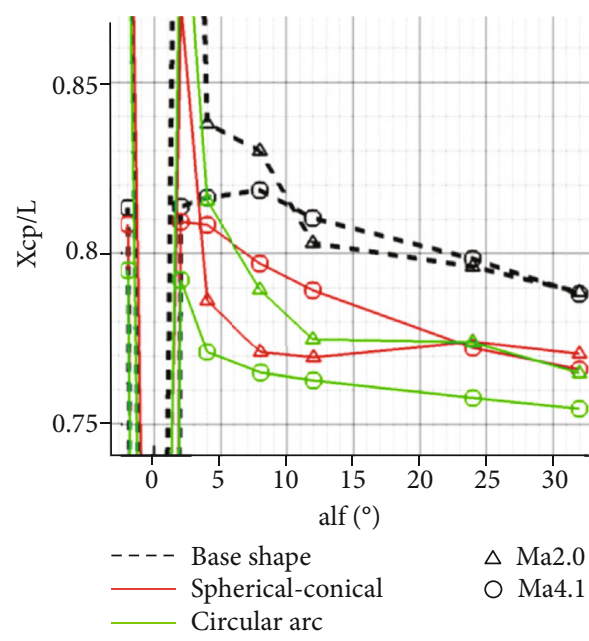

(b)

FiguRE 8: Comparison between supersonic aerodynamic characteristic curves of the Base shape and improved spherical-conical and circular arc noses of the short blunt ascent vehicle. (a) Axial force coefficient. (b) Pressure center coefficient.

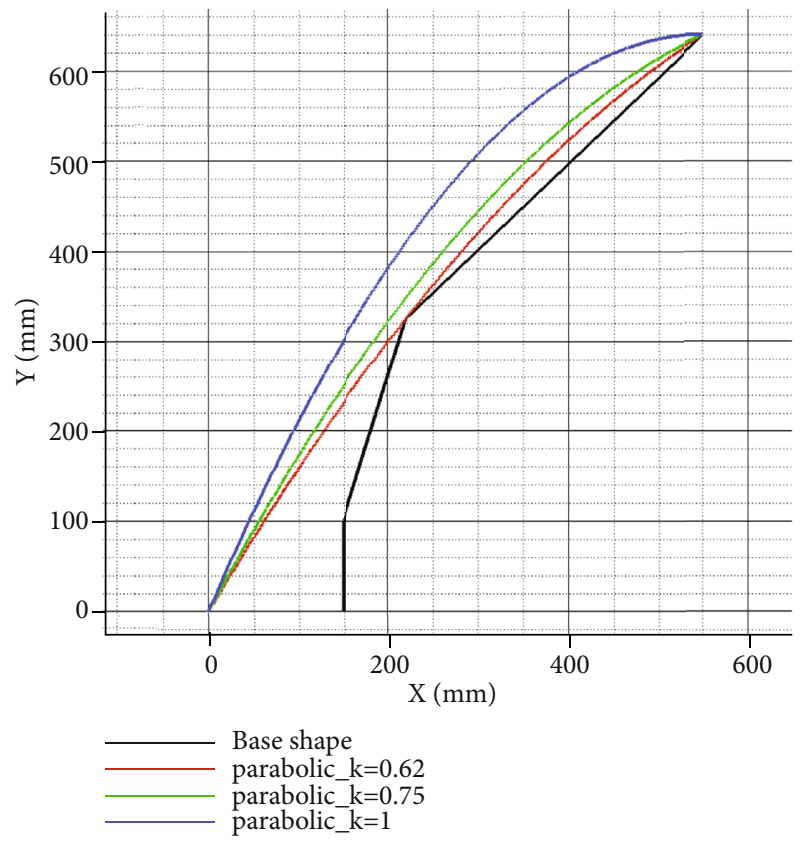

(a)

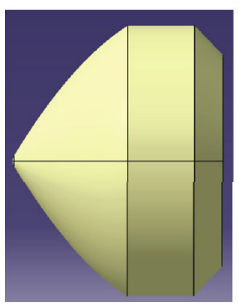

(b)

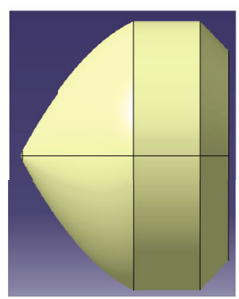

(c)

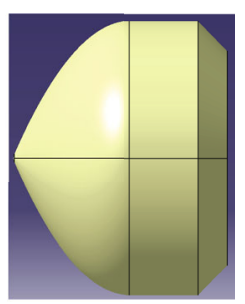

(d)
Figure 9: Optimization results of parabolic noses of the short blunt ascent vehicle. (a) Comparison of optimized shape and configuration constraint contour of three parabolic noses. (b) Parabolic nose with $k=0.62$. (c) Parabolic nose with $k=0.75$. (d) Parabolic nose with $k=1$. improved spherical-conical and circular arc noses at Ma2.0 and Ma4.1 are obtained, as shown in Figure 8. $L$ in the pressure center coefficient $\mathrm{Xcp} / L$ is the total height of the short blunt body. By comparison, the following conclusions can be drawn:

(1) The pressure center coefficient of the Base shape and the two improved noses is less than 0.45 at a small attack angle. The spherical-conical nose has the largest pressure center coefficient, but it is difficult to maintain static stability because the pressure center is too close to the nose

(2) After the Base shape is changed to the sphericalconical or circular arc nose, the CA decreases slightly, but the drag reduction effect of the spherical-conical nose becomes stronger

(3) Compared with that of the Base shape, the zeroattack-angle CA of circular arc nose decreases by about $3.5 \%$ to 1.232 at Ma2.0 and drops by about $0.5 \%$ to 1.083 at Ma4.1, so that the zero-attackangle drag reduction at a large Mach number is weak

(4) The static stability margin of the two improved noses is reduced as compared with that of the Base shape, but the pressure center coefficient is still above 0.7, which makes it easier to achieve static stability

A group of parabolic curves is designed, and $k$ is set as $0.62,0.75$, and 1.0 , respectively. When $k$ is 0.62 , the generatrix passes through the inner contour point. The parabolic shape has a sharp nose so that it is rounded, and the radius of the spherical nose is $50 \mathrm{~mm}$. The results of shape optimization are shown in Figure 9.

Through CFD numerical simulation, the axial force coefficient and pressure center coefficient of the three optimized parabolic noses at Ma2.0 and Ma4.1 are obtained. They are compared with the corresponding parameters of the circular 


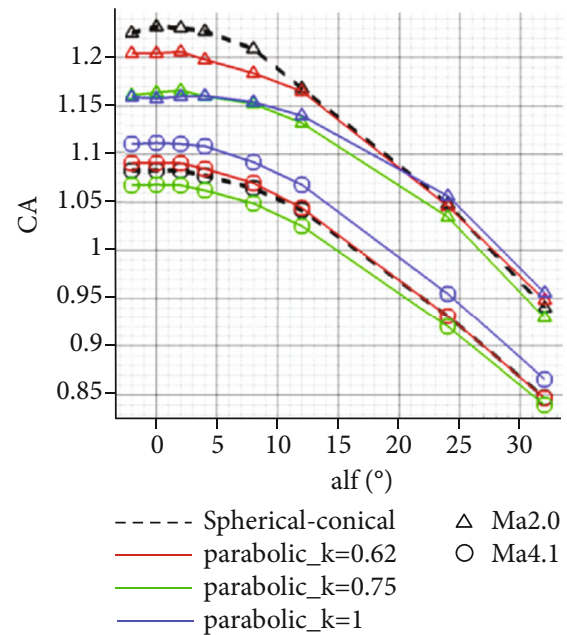

(a)

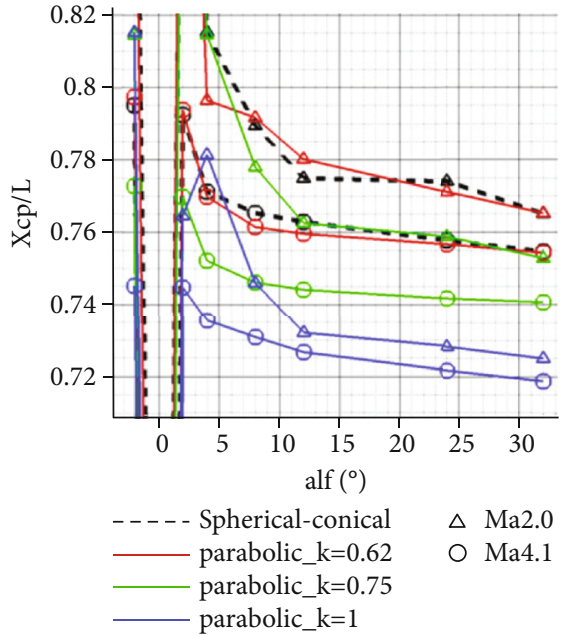

(b)

FIGURE 10: Comparison between supersonic aerodynamic characteristic curves of the circular arc nose and optimized parabolic noses of the short blunt ascent vehicle. (a) Axial force coefficient. (b) Pressure center coefficient.

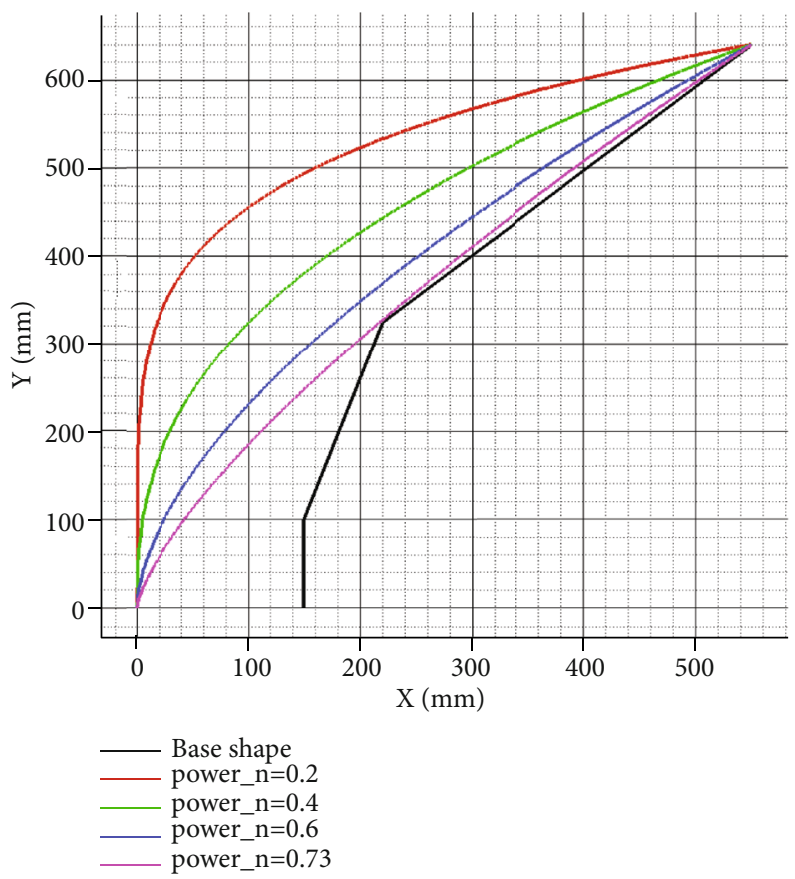

(a)

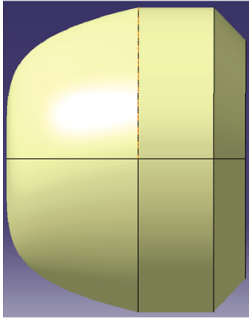

(b)

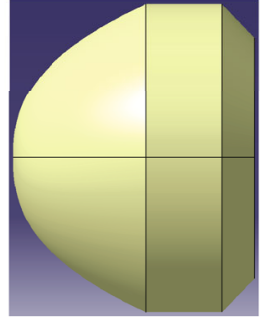

(c)

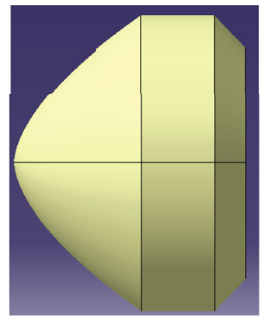

(d)

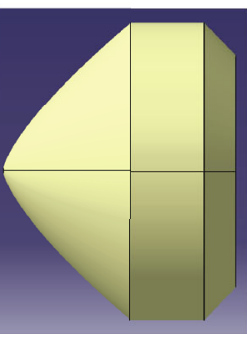

(e)

FIGURE 11: Optimization results of exponential noses of the short blunt ascent vehicle. (a) Comparison of optimized shape and configuration constraint contour of four exponential noses. (b) Exponential nose with $n=0.2$. (c) Exponential nose with $n=0.4$. (d) Exponential nose with $n=0.6$. (e) Exponential nose with $n=0.73$. 


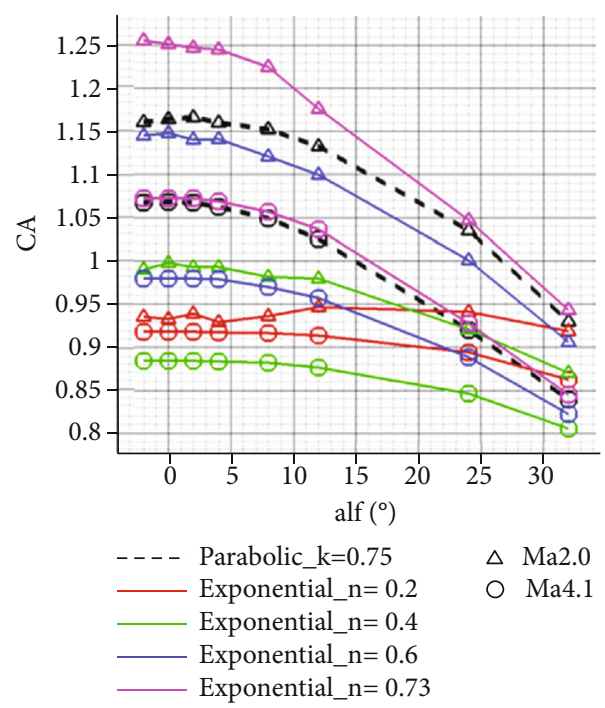

(a)

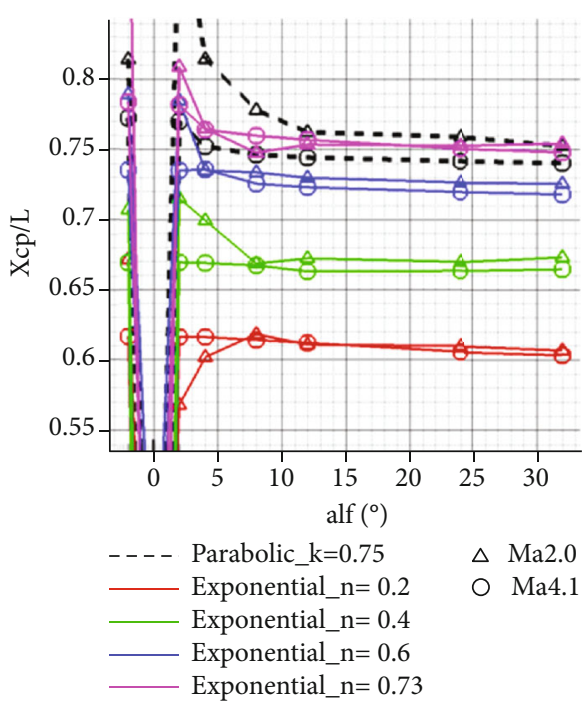

(b)

Figure 12: Comparison between supersonic aerodynamic characteristic curves of the parabolic nose and optimized exponential noses of the short blunt ascent vehicle. (a) Axial force coefficient. (b) Pressure center coefficient.

arc nose under the same conditions, as shown in Figure 10. By comparison, the following conclusions can be drawn:

(1) Compared with the circular arc nose, the parabolic nose has better drag characteristics at a low supersonic velocity; the drag characteristics better than those of the circular arc nose can also be obtained through reasonable $k$ at a large Mach number. Among the three curves with $k=0.62 / 0.75 / 1.0$, when $k$ is 0.75 , the optimal drag characteristics are obtained and are better than those of the circular arc nose under the calculation conditions

(2) Compared with the circular arc nose, the zeroattack-angle CA of the parabolic nose with $k=0.75$ declines by about $5.5 \%$ to 1.164 at Ma2.0 and drops by about $1.4 \%$ to 1.0683 at Ma4.1, so that the zero-attack-angle drag reduction at a large Mach number is weak

(3) The static stability margin of the parabolic nose is less than that of the circular arc nose, but it is easier to ensure static stability

Based on a comprehensive comparison, the aerodynamic performance of the parabolic nose is better than that of the previous two improved noses.

Another group of exponential curves is designed, and $n$ is set as $0.2,0.4,0.6$, and 0.73 , respectively. When $k$ is 0.73 , the generatrix passes through the inner contour point. The optimization results for the exponential nose are shown in Figure 11.

Through CFD numerical simulation, the axial force coefficient and pressure center coefficient of the four optimized exponential noses at Ma2.0 and Ma4.1 are obtained. They are compared with the corresponding parameters of the par- abolic nose with $k=0.75$ under the same conditions, as shown in Figure 12. By comparison, the following conclusions can be drawn:

(1) Compared with the parabolic nose, the exponential nose with $n \leq 0.6$ has better drag characteristics at a low supersonic velocity, and the smaller $n$ will lead to the smaller CA

(2) The CA curves at different Mach numbers approach gradually with the smaller $n$. When $n=0.2$, the difference between the CA curves at Ma2.0 and Ma4.1 is less than $2 \%$ within the range of attack angle $\left(0^{\circ}-5^{\circ}\right)$.

(3) Compared with the parabolic nose with $k=0.75$, the zero-attack-angle CA of the exponential nose $(n=0.2)$ decreases by about $19.8 \%$ to 0.933 at Ma2.0 and drops by about $14.0 \%$ to 0.918 at Ma4.1, with evident drag reduction

(4) The exponential nose with $n=0.4$ is close to that with $n=0.2$ in the supersonic drag reduction. It can be inferred that, when $n=0.2-0.4$, the aerodynamic performance of the exponential nose still requires optimization

(5) The static stability margin of the exponential nose is less than that of the parabolic nose. The minimum pressure center coefficient of the exponential nose with $n=0.2$ is 0.6 so that it is difficult to maintain static stability

The comprehensive comparison demonstrates that the drag reduction by the exponential nose with $n=0.2-0.4$ is visible. Since the pressure center coefficient is higher when $n=0.2$, the in-depth optimization shall be carried out for the exponential nose with $0.2<n \leq 0.4$ in the actual design. 
According to the mechanism analysis based on the flow field analysis, the characteristic that the exponential nose with small $n$ has the most evident drag reduction and the worst static stability can be explained as follows: the smaller $n$ of the exponential nose will lead to the larger bluntness at the crown, but the smaller crown area will lead to the smaller taper of the rear curved surface of the crown. When there is a crown with large bluntness and small area, the detached shock wave will only exist in the crown with a small area, with low intensity; the flow on the surface with a small taper will be smoother, and the pressure and friction on it will be very small. However, the smaller $n$ of the exponential nose will lead to the larger flow transition between the crown and its adjacent surface, so that the pressure will change fast and the pressure center will move forward rapidly. Therefore, the static stability of the exponential nose with small $n$ will become lower.

\section{Conclusions}

The paper analyzed the requirements of supersonic aerodynamic performance of MAV, and the influence laws and numerical values of bus parameters of different types of MAV on supersonic drag characteristics and static stability were calculated and analyzed. Finally, the following conclusions can be drawn:

(1) For slender ascent vehicle, the shape of conical forebody can play a better role in drag reduction, and the drag performance after drag reduction can meet the demand. However, the slender body has poor static stability due to its front center of pressure, and the improvement of forebody shape has little effect on static stability

(2) For the short blunt body riser, the exponential forebody with $0.2<n \leq 0.4$ can greatly improve the resistance performance and reduce the resistance coefficient. Meanwhile, the static stability margin of the short blunt body is easy to meet because the center of pressure at a small angle of attack is closer to the tail end, so the drag reduction design of the forebody shape should be emphasized

Further research will be carried out on the influence of the tail shape optimization of the ascent vehicle on aerodynamic stability, the optimization design of the forebody resistance reduction structure, and the aerodynamic performance, so as to explore more effective methods for reducing energy consumption and improving the bearing capacity of the system of the Mars ascent vehicle.

\section{Conflicts of Interest}

All authors declare no possible conflicts of interests.

\section{Authors' Contributions}

Li Qi completed the conception and compilation of the manuscript. Yuan $\mathrm{Wu}$ completed the aerodynamic layout optimization design of the Mars ascent vehicles. Zhao Rui contributed to the calculation of static aerodynamic data of the Mars ascent vehicles. Wei Haogong performed the data analysis.

\section{Acknowledgments}

This is supported by the Major Planetary Exploration Projects and the National Natural Science Foundation of China 11902025 .

\section{References}

[1] Strategy CM, "The State Council Information Office of the People's Republic of China. China's space actives in 2016," Aerospace China, vol. 1, pp. 10-17, 2017.

[2] A. Karp, B. Nakazono, R. Shotwell et al., "A hybrid mars ascent vehicle design and FY 2016 technology development," in 2017 IEEE Aerospace Conference, pp. 1-10, Big Sky, MT, USA, 2017.

[3] I. J. Dux, J. A. Huwaldt, R. S. McKamey, and J. W. Dankanich, "Mars ascent vehicle gross lift-off mass sensitivities for robotic Mars sample return," in 2011 Aerospace Conference, pp. 1-16, Big Sky, MT, USA, 2011.

[4] B. G. Drake and K. D. Watts, Human exploration of Mars design reference architecture 5.0, Addendum 2, NASA, 2009.

[5] A. A. Gonzales, C. R. Stoker, L. G. Lemke et al., "Mars sample return using commercial capabilities: mission architecture overview," in 2014 IEEE Aerospace Conference, pp. 1-15, Big Sky, MT, USA, 2014.

[6] B. Gardini and A. Santovincenzo, "The Aurora Mars sample return mission," in 54th International Astronautical Congress of the International Astronautical Federation, the International Academy of Astronautics, and the International Institute of Space Law, Bremen, Germany, 2003.

[7] J. S. Martin, Mars engineering model, NASA, 1975.

[8] W. Rong and G. L. Chen, "The characters of deceleration and landing technology on Mars explorer," Spacecraft Recovery \& Remote Sensing, vol. 31, no. 4, pp. 1-6, 2010.

[9] J. V. Bowles, L. C. Huynh, V. M. Hawke, and X. J. Jiang, Mars sample return: Mars ascent vehicle mission and technology requirements, NASA, 2013.

[10] W. Tang, D. W. Jiang, and Y. W. Gui, "Study on generatrix curvetypes of axis-symmetric missiles," Acta Aerodynamica Sinica, vol. 28, no. 2, pp. 218-221, 2010.

[11] R. S. Miao, J. M. Ju, and J. S. Wu, Missile Aerodynamics, National Defense Industry Press, 2006.

[12] B. B. Feng, D. R. Chen, J. D. Wang, and X. T. Yang, "Effect of nose shape on flight dynamics of supersonic vehicles," Flight Dynamics, vol. 30, no. 6, pp. 537-540, 2012.

[13] W. L. Wang, H. Li, F. Liu, and S. Pan, "Research on drag characteristics of supersonic vehicle influenced by different base flows," Computer Simulation, vol. 33, no. 5, pp. 105110, 2016.

[14] P. I. Roe, "Approximate Riemann solvers, parameter vectors, and difference schemes," Journal of Computational Physics, vol. 43, no. 2, pp. 357-372, 1981.

[15] C. Yan, Computational fluid dynamics methods and applications, Beijing University of Aeronautics and Astronautics Press, Beijing, China, 2006. 
[16] A. A. Dyakonov, J. W. MarkSchoenenberger, and V. Norman, "Hypersonic and supersonic static aerodynamics of Mars science laboratory entry vehicle," in 43rd AIAA Thermophysics Conference, p. 2999, New Orleans, LA, 2012.

[17] P. A. Gnoffo, K. J. Weilmuenster, R. D. Braun, and C. I. Cruz, "Influence of sonic-line location on Mars pathfinder probe aerothermodynamics," Journal of Spacecraft and Rockets, vol. 33, no. 2, pp. 169-177, 1996. 